\title{
Antiglycative and anti-inflammatory effects of lipophilized tyrosol derivatives
}

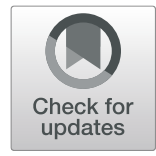

Xiaoqian $\mathrm{Hu}^{1}$, Mingfu Wang ${ }^{2^{*}}$ and Fereidoon Shahidi ${ }^{2,3^{*}}$ (1)

\begin{abstract}
To expand the application of tyrosol, a series of lipophilized tyrosol derivatives were synthesized via esterification of tyrosol with fatty acids of different chain lengths. The antiglycative activity of tyrosol esters so prepared was subsequently examined in the bovine serum albumin/glucose system. A quasi-parabolic shape was observed when the activity was plotted against alkyl chain length. Additionally, the anti-inflammatory effects of these derivatives were evaluated against methylglyoxalinduced inflammation in RAW264.7 cells. The same trend on anti-inflammatory activity was found as in the antiglycation study. The results showed that tyrosol esters with C12:0 and C14:0 were two most efficient ones among all the tested derivatives. Thus, some lipophilized tyrosol derivatives were stronger antiglycative and anti-inflammatory agents compared to the parent compound, tyrosol.
\end{abstract}

Keywords: Tyrosyl esters, Lipophilization, Glycation, Methylglyoxal, Inflammation, Phenolipids

\section{Introduction}

Glycation is a non-enzymatic reaction between reducing sugars and proteins, forming an unstable Schiff base which rearranges to a relatively stable Amadori product (Yan et al. 2003). Glycation occurs in living organisms under normal and pathological conditions. Once Amadori product is formed, it follows a further reaction to produce dicarbonyl intermediates as precursors (Ahmed and Thornalley 2003). Advanced glycation end products (AGEs) are the outcome of glycation process with crosslinked and fluorescent structures (Luevano-Contreras and Chapman-Novakofski 2010). AGEs are found in different types of cells, particularly during aging and chorionic disorders such as diabetes and Alzheimer's disease (Yamagishi and Matsui 2016). Besides, excessive accumulation of AGEs is thought to etiologically contribute to chronic pathologies (Crisostomo et al. 2013; Ramasamy et al. 2005).

Methylglyoxal (MGO), a well-known precursor of AGEs, is generated in cells during oxidation of glucose as well as lipids (Thornalley 2005). MGO has been found in

\footnotetext{
* Correspondence: mfwang@shou.edu.cn; fshahidi@mun.ca

${ }^{2}$ College of Food Science and Technology, Shanghai Ocean University,

Shanghai 201306, China

Full list of author information is available at the end of the article
}

various tissues, where they may be involved in some detrimental processes, particularly under hyperglycemic conditions. MGO may interact with cellular proteins or extracellular matrix proteins to form AGEs, and impair the function of proteins, leading to cellular dysfunction like inflammation response (Cantero et al. 2007; Vulesevic et al. 2016). Therefore, strategies for preventing AGEs formation and AGEs/MGO-induced dysfunction have attracted much interest in recent years. Some polyphenolic compounds have previously been investigated to inhibit the formation of AGEs and show protective effect against AGEs-induced oxidative stress and inflammation (Wang et al. 2016).

Tyrosol (2-(4-hydroxyphenyl) ethanol, TY) is a phenylethanoid present in numerous natural plant sources, particularly in olives and olive oil (Tuck and Hayball 2002). Tyrosol is proven to have beneficial functions related to its antioxidant, antiglycative, cardio-protective and anti-inflammatory properties (Bertelli et al. 2002; Muriana et al. 2017; Zhou et al. 2017). As a phenolic compound, the biological activities of tyrosol depend on both the intake level and bioavailability. Esterification has recently been applied to certain phenolic compounds including tyrosol, in order to improve their hydrophobicity and efficiency in lipid systems (Wang et al. 2016).

(c) The Author(s). 2020 Open Access This article is licensed under a Creative Commons Attribution 4.0 International License, which permits use, sharing, adaptation, distribution and reproduction in any medium or format, as long as you give appropriate credit to the original author(s) and the source, provide a link to the Creative Commons licence, and indicate if changes were made. The images or other third party material in this article are included in the article's Creative Commons licence, unless indicated otherwise in a credit line to the material. If material is not included in the article's Creative Commons licence and your intended use is not permitted by statutory regulation or exceeds the permitted use, you will need to obtain permission directly from the copyright holder. To view a copy of this licence, visit http://creativecommons.org/licenses/by/4.0/. 
Several research efforts have been made on the synthesis of phenolipids from tyrosol and evaluation of their bioactivities. However, these studies have focused primarily on their antioxidant or antimicrobial properties and little is known about their antiglycative and anti-inflammatory properties.

To better understand the application of tyrosol, a series of tyrosol esters were synthesized by esterification of tyrosol with different fatty acids. These fatty acids varied in their chain length from 6 to 18 carbon atoms, including saturated, monounsaturated and polyunsaturated fatty acids. In the present study, we aimed to investigate whether esterification of tyrosol would alter its antiglycative activity via a protein glycation system, and the antiinflammatory effect in an MGO-induced inflammation cell model.

\section{Materials and methods Chemicals}

Tyrosol, bovine serum albumin (BSA), aminoguanidine, Methylglyoxal were purchased from Sigma-Aldrich (St Louis, MO, USA). D-Glucose was purchased from Aladdin (Shanghai, China).

\section{Preparation of tyrosol esters}

Tyrosol esters were synthesized according to the method described by Sun et al. (2018). The same products were also prepared as given here. Briefly, tyrosol and fatty acid of different chain-length and unsaturation degree (Caproic acid, caprylic acid, capric acid, lauric acid, myristic acid, palmitic acid, stearic acid, oleic acid, linolenic acid and eicosapentaenoic acid) were reacted with tyrosol in the presence of Candida antarctica lipase at $40^{\circ} \mathrm{C}$ for $24 \mathrm{~h}$. Any unreacted starting materials, mainly fatty acids, were removed by using a base extraction process. Both routes provided identical products with no side products formed and products were $+99 \%$ pure.

\section{Measurement of total fluorescent AGEs}

AGEs were measured in an in vitro glycation model with or without potential inhibitors. Bovine serum albumin (BSA, $2 \mathrm{mg} / \mathrm{mL}$ ) was co-incubated with D-glucose $(6$ $\mathrm{mg} / \mathrm{L}$ ) in $0.1 \mathrm{M}$ phosphate buffer ( $\mathrm{pH} 7)$. To evaluate inhibition of AGEs, a series of tyrosol esters as well as aminoguanidine (AG), as a positive control, were added. The glycation system was incubated at $37^{\circ} \mathrm{C}$ for 7 days. After incubation, the level of AGEs was recorded by monitoring fluorescence intensity in a multi-mode microplate reader (Synergy 2, Biotek, Winooski, VT, USA), with excitation and emission wavelengths of 355 and $405 \mathrm{~nm}$, respectively.
Cell culture and determination of cell viability

RAW 264.7 macrophages from American Type Culture Collection were cultured in DMEM containing 10\% fetal bovine serum at $37^{\circ} \mathrm{C}$ and $5 \% \mathrm{CO}_{2}$. A cell suspension was prepared and added into a 96-well plate at a concentration of $2 \times 10^{5}$ cells $/ \mathrm{mL}$. To determine the toxicity of MGO, $0-1000 \mu \mathrm{M}$ of MGO was added. To determine the cytoprotective effects of chemicals, the cells were pretreated with AG, tyrosol and tyrosol ester derivatives at a given concentration for $12 \mathrm{~h}$, and then switched to a medium containing $1000 \mu \mathrm{M}$ MGO to induce toxicity, followed by incubation for a further $12 \mathrm{~h}$. Cell number of each well was determined by crystal violet staining.

\section{RNA isolation}

RAW264.7 cells were seeded onto a 12-well plate. When grown to $70 \%$ confluence, cells were treated with $2 \mu \mathrm{M}$ chemicals, including AG, tyrosol and tyrosol esters. After $12 \mathrm{~h}$ of pretreatment, $200 \mu \mathrm{M}$ of MGO were added to cells and incubated for $12 \mathrm{~h}$, which had no effect on cell proliferation or cell death.

The cells were then harvested, and their total RNA was isolated by using TRIzol reagent. The RNA concentration was measured with Nanodrop spectrophotometers (Thermo, Wilmington, DE, USA).

\section{Real-time PCR}

RNA (1 $\mu \mathrm{g})$ was used for reverse transcription which was performed with a SuperScript VILO cDNA synthesis kit (Invitrogen, Carlsbad, CA, USA) with random hexamers, according to the instruction of the manufacturer. Gene expression was determined with real time qPCR using SYBRGreen reagent. Primer sequences are listed in Table 1.

\section{Statistical analysis}

Data were expressed as the mean \pm standard deviation (SD). All statistical analyses were carried out by using Graphpad Prism 5.0 software. Differences with $P<0.05$ were considered to be statistically significant.

Table 1 Primers used for real-time qPCR

\begin{tabular}{lll}
\hline Gene & Forward primer & Reverse primer \\
\hline IL-1 $\boldsymbol{\beta}$ & GCAACTGTTCCTGAACTCAACT & ATCTITTGGGGTCGTCAACT \\
IL-6 & AGCCAGAGTCCTTCAGAGAGAT & GCACTAGGTTTGCCGAGTAGAT \\
iNOS & GGCAGCCTGTGAGACCTITG & GCATTGGAAGTGAAGCGTTTC \\
MIP2 & CTGTCAATGCCTGAAGACC & CCGGGTGTGTTGTTT \\
TNF-a & CACCACGCTCTTCTGTCTACTG & CTTGAGATCCATCGCGTTG \\
$\mathbf{1 8 s}$ & GTAACCCGTTGAACCCCATT & CCATCCAATCGGTAGTAGCG \\
\hline
\end{tabular}




\section{Results}

Inhibitory effect of tyrosol and its esters on glycation The anti-glycative effect of tyrosol has been welldocumented. As previous studies have shown, esterification of EGCG with fatty acids alters its ability for inhibiting glycation (Wang et al. 2016). Therefore, it is expected that tyrosol esters with different fatty acids would likely have different activities compared to the parent molecule. Figure 1 presents the anti-glycative activities of tyrosol and its esters, as evaluated by the change of fluorescent AGEs in the BSA/glucose system. Tyrosol showed an $8.27 \%$ inhibition on total AGEs formation. Different esters with fatty acids enhanced or suppressed the inhibitory effects compared with the parent tyrosol molecule. Among these derivatives, esters of tyrosol with C10:0, C12:0, C14:0 and C18:1 enhanced the inhibition of AGEs formation, compared with the parent tyrosol molecule. Meanwhile esters with C6:0, C8:0, C16:0, c18:0 and C18:1 exhibited lower antiglycative activities than tyrosol. Esters with $\mathrm{C} 18: 3$ had no anti-glycation effects, even stimulating AGEs formation when compared to the control. The relationship of the antiglycative efficiency and the fatty acid chain length was nonlinear, but quasi-parabolic.

\section{Cytoprotective effect of tyrosol and its esters in MGO- treated RAW264.7 cells}

The cytotoxicity of MGO at concentrations of $0-1000$ $\mu \mathrm{M}$ in cells was first examined. As shown in Fig. 2a, MGO showed no significant effect on cell number and morphological change at low concentrations (0$600 \mu \mathrm{M})$. Treatment with MGO at higher concentrations $(600-1000 \mu \mathrm{M})$ caused a cytotoxic effect, acting in a dose-dependent manner. To determine the potential cytoprotective effect, $1000 \mu \mathrm{M}$ MGO was used to induce cell death. Among those compounds which showed inhibitory effect on glycation, tyrosol esters with $\mathrm{C} 12: 0$ and $\mathrm{C} 14: 0$ showed significant cytoprotective activity and the cell viabilities were increased by 29.4 and $30.4 \%$ compared with MGO-treated cells (Fig. 2b).
Inhibition of gene expression of pro-inflammatory cytokine in MGO-treated RAW264.7 cells

RAW264.7 cell line is widely used to study inflammation event under certain conditions and treatment with different chemicals. In this study, the nontoxic concentration, $200 \mu \mathrm{M}$ of MGO was chosen for stimulation of inflammation in RAW264.7 cells. Typical proinflammatory cytokines, such as tumor necrosis factor$\alpha$ (TNF- $\alpha$ ), interleukin-1 $\beta$ (IL-1 $\beta$ ) and interleukin-6 (IL6) were examined on gene expression level to evaluate whether MGO could induce inflammation. Figure 3 shows that with MGO treatment, TNF $\alpha$ and IL-1 $\beta$ mRNA were dramatically evoked $(P<0.01)$, but IL-6 did not significantly increase. Taken together, MGO at 200 $\mu \mathrm{M}$ was able to induce some inflammatory response in RAW264.7 macrophages. To evaluate the antiinflammatory effect of tyrosol esters, the cells were pretreated with them to see whether they could attenuate the inflammation induced by MGO. As shown in Fig. 3a, all tyrosol esters were more effective in reducing TNF $\alpha$ mRNA expression than the parent compound, tyrosol, especially for esters with $\mathrm{C} 12: 0 \quad(56.0 \%$ decrease of MGO-treated group, $P<0.01$ ), C14:0 (51.4\% decrease of MGO-treated group, $P<0.01)$ and C18:1(42.3\% decrease of MGO-treated group, $P<0.05)$. The same trend was observed in IL-1 $\beta$ mRNA expression. Except for esters with $\mathrm{C} 18: 1$, other esters had stronger inhibiting capacity on IL-1 $\beta$ gene expression than tyrosol (Fig. 3b). For IL-6 mRNA, the parent tyrosol showed stronger inhibitory effect $(75.8 \%$ decrease of MGO-treated group, $P<0.01)$ than its esters with different fatty acids (Fig. 3c). Interestingly, although tyrosol ester with C18:3 could not inhibit AGEs formation, as shown in Fig. 1, it could significantly reduce the mRNA expression of some inflammatory cytokines, such as IL-1 $\beta$ and IL-6 (Fig. 4).

\section{Inhibition of iNOS and MIP-2 mRNA in MGO-treated RAW264.7 cells}

In macrophages, chemokines are also involved in inflammation. To systemically evaluate the anti-inflammatory effects of tyrosol esters, other important inflammation mediators including Macrophage Inflammatory Protein

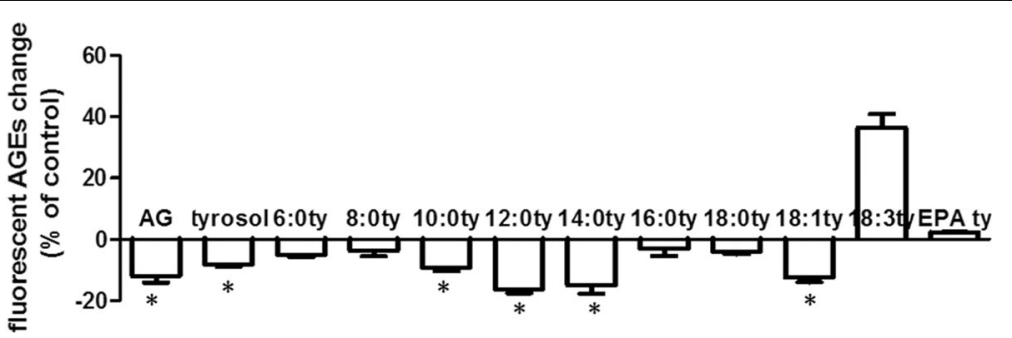

Fig. 1 Inhibitory effect of tyrosol and its esters on glycation. The antiglycation assay was performed in a BSA glucose model with $100 \mu \mathrm{M}$ of aminoguanidine (AG), tyrosol and its esters. Data are means of duplicates from three separate experiments. ${ }^{*} P<0.05$. Ty, tyrosol 

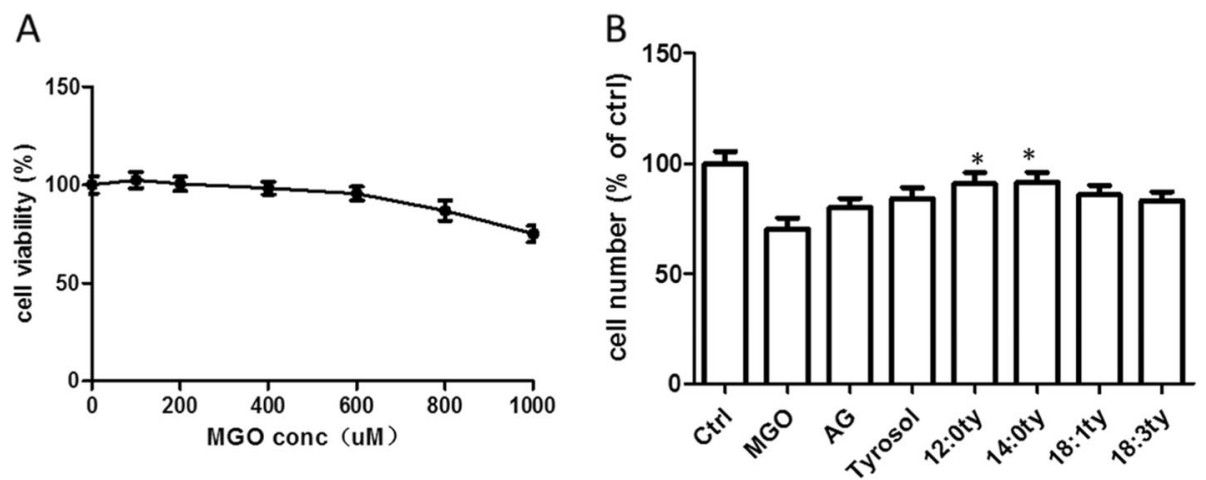

Fig. 2 Cytoprotective effect of tyrosol and its esters in MGO-treated RAW264.7 cells. a Effect of MGO on cell viability was determined at treatment of $0,200,400,600,800$ and $1000 \mu \mathrm{M}$ for $24 \mathrm{~h}$. b Cells were pretreated with $2 \mu \mathrm{M}$ of tyrosol and tyrosol esters for $12 \mathrm{~h}$, and then incubated with 1000uM of methylglyoxal (MGO) for another $12 \mathrm{~h}$. Data expressed as means of three experiments. ${ }^{*} P<0.05$, vs. control. Ty, tyrosol; Ctrl, control

2 (MIP-2) and inducible nitric oxide synthase (iNOS) were also analyzed. MIP-2, a chemokine, plays a key role in the recruitment and activation of inflammatory cells under certain conditions (De Filippo et al. 2008). In the present study, MGO did not alter the expression of MIP-2 mRNA. Tyrosol and its esters decreased MIP-2 mRNA moderately, only esters with C14:0 showed a significant inhibitory effect on MIP-2 expression (46.8\% decrease of MGO-treated group, $P<0.05)$. Nitric oxide
(NO) is a signaling molecule which mediates many aspects of inflammatory responses. iNOS is the principal enzyme involved in $\mathrm{NO}$ production, responsible for high-level NO synthesis (Förstermann and Sessa 2012). Therefore, iNOS mRNA was also measured and found to be significantly triggered (3.26-fold, $P<0.05$ ) by MGO treatment. Tyrosol and all its esters significantly reduced iNOS mRNA expression to varying degrees. Among them, tyrosol esterified to $\mathrm{C} 12: 0$ and $\mathrm{C} 14: 0$ was

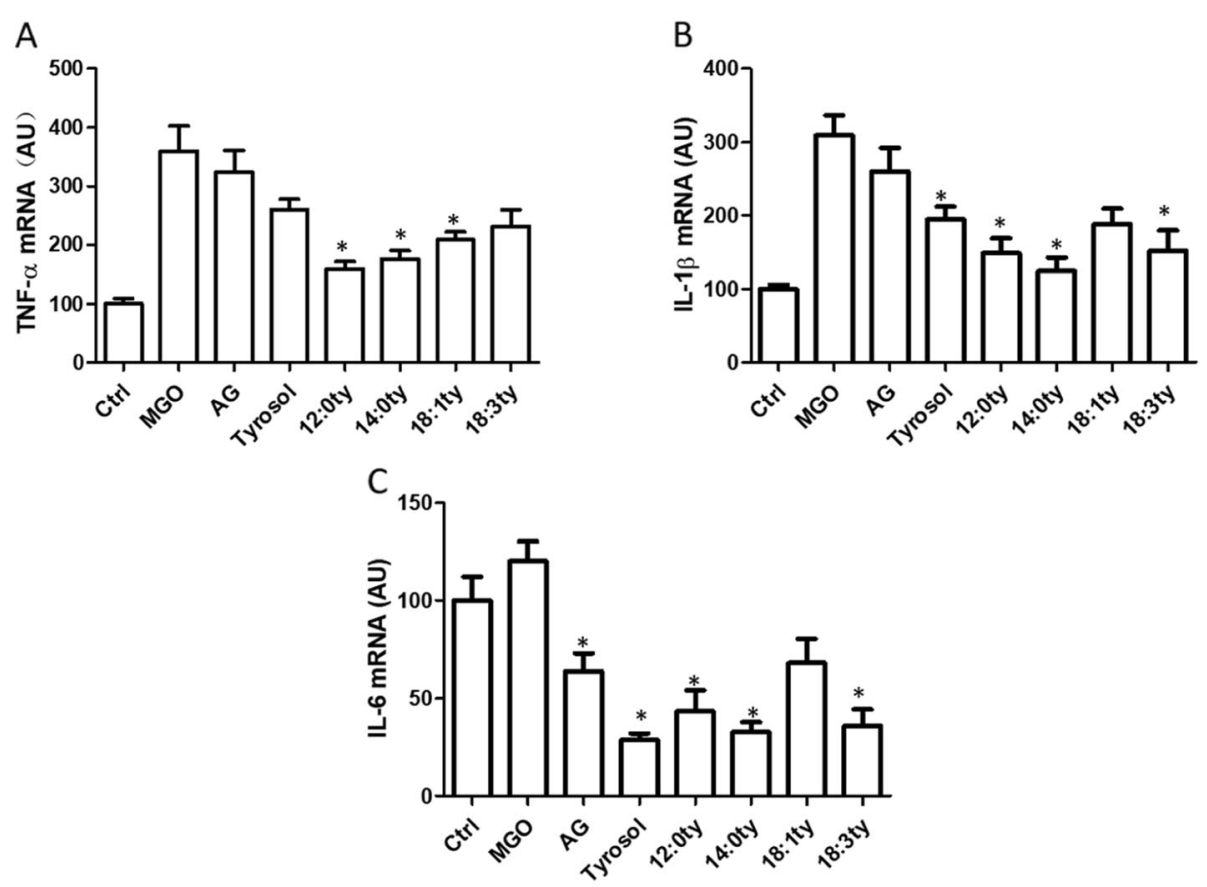

Fig. 3 Tyrosol and its esters inhibited the gene expression of pro-inflammatory cytokines in MGO-treated RAW264.7 cells. Cells are pretreated with $2 \mu \mathrm{M}$ of tyrosol and tyrosol esters for $12 \mathrm{~h}$, then treated with $200 \mathrm{uM} \mathrm{MGO}$ for another $24 \mathrm{~h}$. The total RNAs were transcribed and amplified by qPCR. The mRNA expression of TNF- $a(\mathbf{a}), I L-1 \beta$ and IL-6 (c) were normalized by $18 \mathrm{~s}$ with control. Data expressed as means of triplicates from three experiments. ${ }^{*} P<0.05$, vs. control. Ty, tyrosol; Ctrl, control 

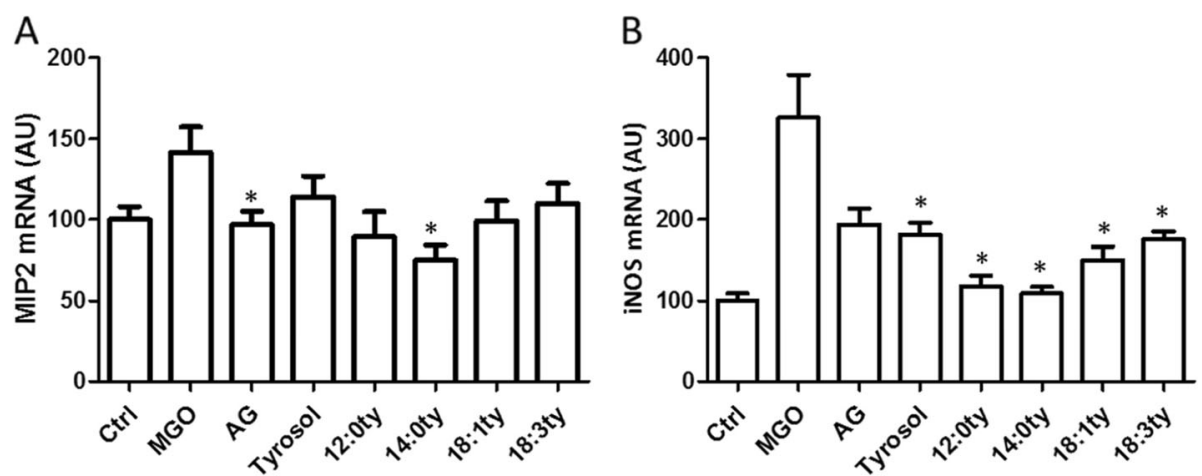

Fig. 4 Tyrosol and its esters inhibited iNOS (a) and MIP-2 (b) gene expression of in MGO-treated RAW264.7 cells. Cells are pretreated with $2 \mu M$ of tyrosol and tyrosol esters for $12 \mathrm{~h}$, then treated with $200 \mathrm{uM} \mathrm{MGO}$ for another $24 \mathrm{~h}$. The total RNAs were transcribed and amplified by qPCR. The data were normalized by $18 \mathrm{~s}$ with control. Data expressed as means of triplicates from three experiments. ${ }^{*} P<0.05$, vs. control. Ty, tyrosol; Ctrl, control

more effective than tyrosol, with 63.9 and $66.6 \%(P<$ $0.01)$ decrease of MGO-treated group, respectively.

\section{Discussion}

Olive and its products are an integral part of the Mediterranean diet and might confer health benefits due to the inclusion of monounsaturated fatty acids and polyphenols. Tyrosol is one of the most abundant phenolic compounds found in olive oil (Richard et al. 2011) and the presence of tyrosol esters in olive oil was more recently documented (Lee et al. 2016). Furthermore, the esters of tyrosol were found to undergo hydrolysis in rat gut sac model, hence releasing tyrosol to render its effects (Yin et al. 2018). However, the biological functions of lipophilic tyrosol esters are not yet well elucidated. Thereby, a series of tyrosol fatty acid esters with different chain length from $\mathrm{C} 6$ to $\mathrm{C} 18$ were synthesized and their antiglycative and anti-inflammatory effects investigated for the first time to clarify the relationship of structure (such as carbon number and degree of unsaturation) and their bioactivities.

Concerning the antiglycative activities, esters of tyrosol with C10:0, C12:0, C14:0 and C18:1 were most effective in inhibiting AGEs formation compared with the parent tyrosol. In the present study, a quasi-parabolic shape was displayed for the whole series of tyrosyl esters, when activity was plotted against alkyl chain length. This phenomenon is also known as cut-off effect, which means the efficiency of the compounds increases concomitantly with the increase of their hydrophobic parts up to a certain chain length and then begins to decrease (Costa et al. 2015). Similar trends were also reported studying the structure-activity relationship of other phenolipids with antioxidant capability. A parabolic dependency was observed in an antioxidant experiment with emulsion systems for chlorogenic acid alkyl esters, where phenolic dodecyl ester exerted the maximum antioxidant activity (Laguerre et al. 2009). In another study with rosmarinic acid alkyl esters, the maximum antioxidant activity was found in the corresponding phenolic octyl ester (Panya et al. 2012). Oh and Shahidi (2018) found that resveratrol octyl ester showed the highest antioxidant activity in bulk oil system.

MGO is generated by glycation during cell metabolism, such as oxidation of glucose and lipids, and MGO can also be formed in our foods, particularly in thermally processed products and fermented foods. This dicarbonyl compound is very reactive and causes protein crosslinking, ultimately leading to the formation of AGEs (Baynes and Thorpe 2000; Cantero et al. 2007). Abnormal accumulation of MGO in the body evokes glycative stress, which might cause carbonylative damage to lipids, proteins and DNA and lead to cell and tissue dysfunction. One of the biological consequences of glycative stress is chronic inflammation (Lin et al. 2016; Vulesevic et al. 2016). Recent studies indicate that blocking of AGEs formation by antiglycative agents is an efficient means to prevent the dysfunction caused by AGEs. Since tyrosyl esters exerted good potentials on inhibiting AGEs formation, it is very likely that these compounds would alleviate inflammation induced by glycative stress.

The anti-inflammatory effect of phenolic esters has been documented. Zhong et al. (2012) and Oh et al. (2019) found the anti-inflammatory potentials of EGCG esters and resveratrol esters, respectively, in macrophages. In this context, it was hypothesized that tyrosol derivatives would show anti-inflammatory effect. In fact, the anti-inflammatory effect of tyrosol has been previously reported by Lu et al. (2013); $0.3 \mathrm{mM}$ tyrosol reduced both gene expression and secretion of proinflammatory cytokines, including TNF- $\alpha$, IL- $1 \beta$ and IL-6 in LPS-induced RAW264.7 cells. However, our data showed that tyrosol was inefficient to decrease TNF- $\alpha$ mRNA expression, only effective on IL-1 $\beta$ and IL-6. The 
different concentrations tested could explain the variance. Although only a few esters with good antiglycative potential were examined in cell model, the same trend of anti-inflammatory activity as antiglycation was observed. Taking all indicators into account, C12:0 and C14:0 tyrosol esters were most efficient in cell survival or improvement of MGO-induced inflammation among the tested tyrosol esters. It is interesting to note that tyrosol ester with C18:3 was not able to inhibit AGEs formation, but it significantly reduced inflammation induced by MGO. The anti-inflammatory effect of these tyrosol esters might rely on other intracellular mechanisms, like altering AGEs signaling. Part of the glycated proteins will direct to proteolysis for losing their function, and some of them may bind to cell surface receptors, like receptor for AGEs (RAGE). RAGE mediates the intracellular signaling of AGEs, activates certain inflammatory regulators and results in inflammation response (Ott et al. 2014). Tyrosol ester with C18:3 probably acts through reducing RAGE expression or blocking its signaling. Further studies are needed to clarify the underlying mechanism.

\section{Conclusion}

The effects of tyrosyl esters with different fatty acid chain length on antiglycation and anti-inflammation activity were examined. Based on the data obtained for AGEs formation, the medium chain derivatives (C10:0 tyrosol ester and C12:0 tyrosol ester) and the C14:0 tyrosol ester exhibited good antiglycation effects. The anti-inflammation activity of tyrosol derivatives showed a similar tendency to that observed on glycation. Tyrosyl esters with C12:0 and C14:0 were most efficient in improving MGO-induced inflammation. Thus, the lipophilized tyrosol esters may have potential use as antiglycation and anti-inflammation agents in food and pharmaceutical applications.

\section{Abbreviations}

AGEs: Advanced glycation end products; MGO: Methylglyoxal; TY: Tyrosol; BSA: Bovine serum albumin; AG: Aminoguanidine; TNF-a: Tumor necrosis factor- a; IL: Interleukin; MIP2: Macrophage Inflammatory Protein 2; iNOS: Inducible nitric oxide synthase; RAGE: Receptor for AGEs

\section{Acknowledgements}

Not applicable.

\section{Authors' contributions}

X. Hu, M.Wang. and F. Shahidi conceived and planned the experiments, X. Hu carried out the experiments and wrote the manuscript. M.Wang. and F. Shahidi revised the manuscript. The author(s) read and approved the final manuscript.

\section{Funding}

The research work was supported, in part or in whole, by National Natural Science Foundation of China (Grant no. 31671821) and Open Research Fund of State Key Laboratory of Molecular Vaccinology and Molecular Diagnostics (Grant no. SKLVD2020KF06) and in part by the Natural Science and Engineering Research Council (NSERC) of Canada.
Availability of data and materials

All needed data is presented in this manuscript.

Ethics approval and consent to participate

Not applicable.

Consent for publication

All authors consent to the publication of the manuscript.

\section{Competing interests}

The authors declare that they have no competing interests.

\section{Author details}

${ }^{1}$ School of Public Health, Xiamen University, Xiamen 361102, Fujian, China. ${ }^{2}$ College of Food Science and Technology, Shanghai Ocean University, Shanghai 201306, China. ${ }^{3}$ Department of Biochemistry, Memorial University of Newfoundland, St. John's, NL A1B 3X9, Canada.

Received: 1 October 2020 Accepted: 11 November 2020

Published online: 05 December 2020

\section{References}

Ahmed, N., \& Thornalley, P. J. (2003). Quantitative screening of protein biomarkers of early glycation, advanced glycation, oxidation and nitrosation in cellular and extracellular proteins by tandem mass spectrometry multiple reaction monitoring. Biochemical Society Transactions, 31(Pt 6), 1417-1422.

Baynes, J. W., \& Thorpe, S. R. (2000). Glycoxidation and lipoxidation in atherogenesis. Free Radical Biology \& Medicine, 28(12), 1708-1716.

Bertelli, A. A., Migliori, M., Panichi, V., Longoni, B., Origlia, N., Ferretti, A., Giovannini, L. (2002). Oxidative stress and inflammatory reaction modulation by white wine. Annals of the New York Academy of Sciences, 957, 295-301.

Cantero, A. V., Portero-Otin, M., Ayala, V., Auge, N., Sanson, M., Elbaz, M., ... NegreSalvayre, A. (2007). Methylglyoxal induces advanced glycation end product (AGEs) formation and dysfunction of PDGF receptor-beta: implications for diabetic atherosclerosis. The FASEB Journal, 21(12), 3096-3106.

Costa, M., Losada-Barreiro, S., Paiva-Martins, F., Bravo-Diaz, C., \& Romsted, L. S. (2015). A direct correlation between the antioxidant efficiencies of caffeic acid and its alkyl esters and their concentrations in the interfacial region of olive oil emulsions. The pseudophase model interpretation of the "cut-off" effect. Food Chemistry, 175, 233-242.

Crisostomo, J., Matafome, P., Santos-Silva, D., Rodrigues, L., Sena, C. M., Pereira, P., \& Seica, R. (2013). Methylglyoxal chronic administration promotes diabeteslike cardiac ischaemia disease in Wistar normal rats. Nutrition, Metabolism, and Cardiovascular Diseases, 23(12), 1223-1230.

De Filippo, K., Henderson, R. B., Laschinger, M., \& Hogg, N. (2008). Neutrophil chemokines $\mathrm{KC}$ and macrophage-inflammatory protein-2 are newly synthesized by tissue macrophages using distinct TLR signaling pathways. Journal of Immunology, 180(6), 4308-4315.

Förstermann, U., \& Sessa, W. C. (2012). Nitric oxide synthases: regulation and function. European Heart Journal, 33(7), 829-837.

Laguerre, M., Giraldo, L. J., Lecomte, J., Figueroa-Espinoza, M. C., Barea, B., Weiss, J., Villeneuve, P. (2009). Chain length affects antioxidant properties of chlorogenate esters in emulsion: the cutoff theory behind the polar paradox. Journal of Agricultural and Food Chemistry, 57(23), 11335-11342.

Lee, Y.Y., Crausle, C., Wang, H., Leung, H.H., Vercauteran, J., Galano, J.M., Oger, C. Durand, T., Wan, J.M., and Lee, J.C.Y. (2016). Extra vigin olive oil induced ployunsaturated fatty acid and cholesterol oxidation in rodent liver: is tis accounted for hydroxytyrosol-fatty acid conjugation? Chemical Research in Tioxicilogy, https://doi.org/10.1021/acschemrestox.6b00214.

Lin, J. A., Wu, C. H., Lu, C. C., Hsia, S. M., \& Yen, G. C. (2016). Glycative stress from advanced glycation end products (AGEs) and dicarbonyls: an emerging biological factor in cancer onset and progression. Molecular Nutrition \& Food Research, 60(8), 1850-1864.

Lu, J., Huang, G., Wang, Z., Zhuang, S., Xu, L., Song, B., ... Guan, S. (2013). Tyrosol exhibits negative regulatory effects on LPS response and endotoxemia. Food and Chemical Toxicology, 62, 172-178.

Luevano-Contreras, C., \& Chapman-Novakofski, K. (2010). Dietary advanced glycation end products and aging. Nutrients, 2(12), 1247-1265.

Muriana, F. J. G., Montserrat-de la Paz, S., Lucas, R., Bermudez, B., Jaramillo, S., Morales, J. C., ... Lopez, S. (2017). Tyrosol and its metabolites as antioxidative 
and anti-inflammatory molecules in human endothelial cells. Food \& Function, 8(8), 2905-2914.

Oh, W. Y., Chiou, Y. S., Lee, P. S., Pan, M. H., \& Shahidi, F. (2019). Lipophilized resveratrol affects the generation of reactive nitrogen species in murine macrophages and cell viability of human cancer cell lines. Journal of Food Bioactives, 7, 73-77.

Oh, W. Y., \& Shahidi, F. (2018). Antioxidant activity of resveratrol ester derivatives in food and biological model systems. Food Chemistry, 261, 267-273.

Ott, C., Jacobs, K., Haucke, E., Navarrete Santos, A., Grune, T., \& Simm, A. (2014). Role of advanced glycation end products in cellular signaling. Redox Biology, 2, 411-429.

Panya, A., Laguerre, M., Bayrasy, C., Lecomte, J., Villeneuve, P., McClements, D. J., \& Decker, E. A. (2012). An investigation of the versatile antioxidant mechanisms of action of rosmarinate alkyl esters in oil-in-water emulsions. Journal of Agricultural and Food Chemistry, 60(10), 2692-2700.

Ramasamy, R., Vannucci, S. J., Yan, S. S., Herold, K., Yan, S. F., \& Schmidt, A. M. (2005). Advanced glycation end products and RAGE: A common thread in aging, diabetes, neurodegeneration, and inflammation. Glycobiology, 15(7), 16R-28R.

Richard, N., Arnold, S., Hoeller, U., Kilpert, C., Wertz, K., \& Schwager, J. (2011). Hydroxytyrosol is the major anti-inflammatory compound in aqueous olive extracts and impairs cytokine and chemokine production in macrophages. Planta Medica, 77(17), 1890-1897.

Sun, Y., Zhou, D., \& Shahidi, F. (2018). Antioxidant properties of tyrosol and hydroxytyrosol saturated fatty acid esters. Food Chemistry, 245, 1262-1268.

Thornalley, P. J. (2005). Dicarbonyl intermediates in the maillard reaction. Annals of the New York Academy of Sciences, 1043, 111-117.

Tuck, K. L., \& Hayball, P. J. (2002). Major phenolic compounds in olive oil: metabolism and health effects. The Journal of Nutritional Biochemistry, 13(11), 636-644.

Vulesevic, B., McNeill, B., Giacco, F., Maeda, K., Blackburn, N. J., Brownlee, M., .. Suuronen, E. J. (2016). Methylglyoxal-induced endothelial cell loss and inflammation contribute to the development of diabetic cardiomyopathy Diabetes, 65(6), 1699-1713.

Wang, M., Zhang, X., Zhong, Y. J., Perera, N., \& Shahidi, F. (2016). Antiglycation activity of lipophilized epigallocatechin gallate (EGCG) derivatives. Food Chemistry, 190, 1022-1026.

Yamagishi, S., \& Matsui, T. (2016). Pathologic role of dietary advanced glycation end products in cardiometabolic disorders, and therapeutic intervention. Nutrition, 32(2), 157-165.

Yan, S. F., Ramasamy, R., Naka, Y., \& Schmidt, A. M. (2003). Glycation, inflammation, and RAGE: a scaffold for the macrovascular complications of diabetes and beyond. Circulation Research, 93(12), 1159-1169.

Yin, F., Hu, X., Zhou, D., Ma, X., Tian, X., Huo, X., Rakariyathan, Shahidi, F., and Zhu, B. (2018). Hydrolysis and transport of tyrosol acyl esters in rat intestine. Journal of Agricultural and Food Chemistry 66(47), 125-21-12526.

Zhong, Y., Chiou, Y. S., Pan, M. H., \& Shahidi, F. (2012). Anti-inflammatory activity of lipophilic epigallocatechin gallate (EGCG) derivatives in LPS-stimulated murine macrophages. Food Chemistry, 134, 742-748.

Zhou, D. Y., Sun, Y. X., \& Shahidi, F. (2017). Preparation and antioxidant activity of tyrosol and hydroxytyrosol esters. Journal of Functional Foods, 37, 66-73.

\section{Publisher's Note}

Springer Nature remains neutral with regard to jurisdictional claims in published maps and institutional affiliations. 